\title{
Activation of hepatic natural killer cells and control of liver-adapted lymphoma in the murine model of cytomegalovirus infection
}

\author{
Katja C. Erlach • Verena Böhm • Martin Knabe • \\ Petra Deegen · Matthias J. Reddehase · Jürgen Podlech
}

Received: 13 February 2008 / Published online: 29 February 2008

(C) Springer-Verlag 2008

\begin{abstract}
Hematopoietic stem cell transplantation (HSCT) is a promising therapeutic option against hematopoietic malignancies. Infection with cytomegalovirus (CMV) and tumor relapse are complications that limit the success of HSCT. In theory, CMV infection can facilitate tumor relapse and growth by inhibiting "graft take" and reconstitution of the immune system or by inducing the secretion of tumor cell growth-promoting cytokines. Conversely, one can also envisage an anti-tumoral effect of CMV by cytopathic/oncolytic infection of tumor cells, by inducing the secretion of death ligands for tumor cell apoptosis, and by the activation of systemic innate and adaptive immunity. Here we will briefly review the current knowledge about tumor control in a murine model of CMV infection and liver-adapted B cell lymphoma, with a focus on a putative implication of $\mathrm{CD} 49^{+} \mathrm{NKG} 2 \mathrm{D}^{+}$hepatic natural killer cells.
\end{abstract}

Keywords Apoptosis · Cytomegalovirus · Liver . Lymphoma $\cdot$ Natural killer cells

There are inclusions in this text reproduced or recombined from previous articles published in the Journal of Virology (American Society for Microbiology), which are cited in the reference section.

K. C. Erlach $(\bowtie) \cdot$ V. Böhm $\cdot$ M. Knabe $\cdot$ P. Deegen

M. J. Reddehase $\cdot$ J. Podlech $(\bowtie)$

Institute for Virology, Johannes Gutenberg University,

Obere Zahlbacher Strasse 67, Hochhaus am Augustusplatz,

55131 Mainz, Germany

e-mail: podlech@uni-mainz.de

J. Podlech

e-mail: erlach@uni-mainz.de

\section{Introduction and clinical background}

Allogeneic hematopoietic stem cell transplantation (alloHSCT) combined with donor lymphocyte infusion (DLI) is a promising therapeutic option against hematologic malignancies and is currently the only curative option against forms of leukemias that are not eradicated by conventional antitumoral therapies but result in minimal residual disease/ leukemia (MRD/L) with a high risk of tumor relapse $[1,2]$. Control of chronic myeloid/myelogenous leukemia (CML) by allo-HSCT/DLI is impressive while more aggressive acute leukemias still pose a problem [3]. The therapeutic principle of allo-HSCT/DLI is tumor remission by an immunological graft versus leukemia $(\mathrm{GvL})$ effect mediated by donor lymphocytes. While $\mathrm{T}$ cells specific for polymorphic or heterosomal minor histocompatibility antigens and for non-polymorphic leukemia antigens are the principal cellular mediators of the GvL effect in HLA-matched HSCT/DLI, natural killer cells are critically involved in HLA-incompatible/haploidentical $\mathrm{T}$ cell-depleted HSCT $[4,5]$. However, the success of HLA-matched allo-HSCT/ DLI is limited by graft versus host disease (GvHD) mediated by the alloreactive donor $\mathrm{T}$ cells attacking host tissues. Although T-cell depletion would be a most effective measure for preventing GvHD, this would abolish the desired GvL effect and delay the immunological reconstitution, thereby exposing the immunocompromised patient to the risks of tumor relapse and infections, in particular of reactivated cytomegalovirus (CMV) infection resulting in CMV disease with interstitial pneumonia representing a frequent and severe manifestation $[6,7]$.

Remarkably little is known about a possible interference between CMV infection and tumor relapse. CMV could possibly promote tumor relapse and growth by inhibiting hematopoietic reconstitution and thus also the GvL effect 
or, alternatively, could by itself exert an infection versus leukemia effect, either directly by cytopathic infection of malignant cells of hematopoietic lineages or indirectly by induction of innate and adaptive immunity targeting the tumor cells in a bystander mode. Specifically, cell-bound or secreted death ligands could mediate apoptosis of tumor cells expressing death receptors.

For human CMV (hCMV), a myelosuppressive effect inhibiting hematopoiesis is long known [8], and as shown by the group of Beverly Torok-Storb in vitro in long-term bone marrow cell cultures, hCMV may cause a graft failure by different mechanisms [9]. While 8 out of 20 recent clinical isolates analyzed by this group were found to be myelotropic and to directly inhibit the growth of myeloid, erythroid, and multipotent hematopoietic lineage progenitor cells, laboratory strain Ad169 as well as the majority of recent clinical isolates inhibited in vitro hematopoiesis indirectly by infection of the stromal compartment that is essential for hematopoiesis by forming a network providing niches for stem cell and progenitor cell engraftment and that delivers cytokines, so-called hemopoietins, supporting proliferation and differentiation of hematopoietic cells. Clearly, if these mechanisms apply also to the in vivo situation, CMV infection might be expected to promote tumor relapse after HSCT due to an impaired hematopoietic reconstitution associated with an insufficient GvL effect. However, one can predict a difference between clinical isolates depending upon their cell tropism. Whereas myelotropic isolates may also inhibit malignant hematopoietic lineage cells by cell infection, tumor cells that no longer depend on stromal contact and on hemopoietins are unlikely to be targeted by the majority of the clinical isolates and are rather promoted in their growth by the inhibition of the normal, stroma- and hemopoietin-dependent hematopoiesis and immunological reconstitution after HSCT. Nonetheless, a beneficial role for hCMV infection has been reported for patients with adult T-cell leukemia/ lymphoma [10,11], which might reflect a bystander control of tumor growth by death ligands and other mechanisms of innate and adaptive immunity triggered by the infection.

\section{Murine model of CMV-associated hematopoietic deficiency}

Since CMVs are highly species-specific, hCMV cannot be studied in animal models (see the contribution by Powers et al. in this issue of MMI). The infection of mice with murine CMV (mCMV) is a particularly versatile model as it allows in vivo studies with genetically engineered hosts as well as viruses for elucidating mechanisms and for providing "proof of concept" (for reviews, see [12, 13]). However, one must keep in mind that mCMV and hCMV differ genetically as a result of perfect adaptation to their respective host. Nevertheless, as discussed in greater detail recently [13], the lack of an animal model for hCMV is not really a disadvantage, because any heterologous model would not reflect host-virus adaptation by co-evolution. Thus, principles analyzed in mice infected with mCMV may be closer to the principles operative in humans infected with hCMV than the genetic difference between the viruses might suggest, simply because the hosts differ as well. Furthermore, as discussed above for the mechanism of myelosuppression by hCMV, different clinical isolates of hCMV can also differ significantly, genetically as well as in their cell type tropism and other pathogenetic properties [9], (for reviews, see [14, 15]).

For evaluating the suitability of the mCMV model in studying CMV-mediated inhibition of hematopoiesis and reconstitution after HSCT, the effect of mCMV strain Smith (mCMV-Smith) on the in vitro hematopoiesis was studied in long-term bone marrow cell cultures. Notably, mCMVSmith resembled hCMV Ad169 and the majority of recent clinical isolates of hCMV in that inhibition of hematopoiesis was caused by the infection of the supporting stroma, whereas the myeloid lineage-committed granulocyte-macrophage progenitor cells were not directly targeted and could be rescued from infected cultures by transfer to uninfected stromal cell layers $[16,17]$. Thus, in this particular respect, mCMV-Smith is a model for most but not all isolates of hCMV.

An in vivo model of sublethal hematoablative treatment of BALB/c mice by 5-6 Gy total body $\gamma$-irradiation showed that mCMV infection prevents the autoreconstitution of bone marrow with consequent bone marrow aplasia, pancytopenia, disseminated infection, and lethal multiple-organ CMV disease [18, 19]. Likewise, mCMV infection also led to bone marrow aplasia, also referred to as aplastic anemia (CMV-AA), in the model of heterosomal minor histocompatibility antigen-mismatched HSCT using male (XY) $\mathrm{BALB} / \mathrm{c}$ mice as donors and female (XX) BALB/c mice as recipients, thus creating $\mathrm{XY}-\mathrm{XX}$ chimeras with male hematopoietic cells colonizing female bone marrow stroma [20, 21]. PCR specific for the testes-determining gene $t d y$ located on the Y-chromosome thus allowed to distinguish between cells of the hematopoietic and the stromal compartment. In essence, these studies revealed that mCMV infection leads to a "graft failure" by inhibiting the engraftment of transplanted bone marrow stem and progenitor cells in the infected bone marrow stroma of the experimental HSCT recipients [20-22]. Notably, infection of the stroma was barely productive with a high ratio of IE1-positive stromal cells not proceeding to significant viral DNA replication [20]. Accordingly, the architecture of the stroma was not obviously disrupted. The graft failure was rather explained by a functional deficiency of the stroma in the production of 
essential hemopoietins, including stem cell factor (SCF) and granulocyte colony stimulating factor (G-CSF) [20]. It is important to note, however, that an engraftment sufficient for the reconstitution of protective, antiviral CD8 T cells, and thus for survival, was enforced by high numbers of transplanted bone marrow cells, namely $>10^{6}$ cells [21].

One possibility to be discussed is a protective effect of bone marrow cells against CMV-mediated stromal deficiency. Alternatively, high cell numbers could increase the probability that still intact "niches" supporting hematopoiesis get occupied. Evidence for the latter explanation was provided by serial HSCT using infected XY-XX chimeras as HSCT donors. Even after clearance of the infection of the stroma, the repopulation potential of bone marrow cells derived from these donors was found to be impaired, suggesting a reduced number of pluripotent hematopoietic stem cells as a result of the past infection [22]. Altogether, these models suggested that mCMV might promote tumor relapse after HSCT by inhibiting the reconstitution of antitumoral immunity.

A further mechanism discussed in the literature to explain CMV-mediated myelosuppression and pertinent to a possible tumor-suppressive effect is the upregulation of Fas on hematopoietic progenitor cells by mCMV, which makes them susceptible to Fas-mediated apoptosis [23]. If this applies also to malignant cells of hematopoietic origin, CMV infection might rather support leukemia/lymphoma remission.

\section{Murine model of lymphoma growth in the presence of CMV: model design}

The data discussed above have provided reasonable arguments in both directions: predicting tumor relapse or tumor remission by CMV. We therefore extended the established $\mathrm{BALB} / \mathrm{c}$ model of mCMV infection in the setting of syngeneic experimental HSCT by intravenous transfer of the liver-adapted clonal variant E12E of the BALB/c-derived B cell lymphoma A20 (Fig. 1) [24, 25]. Unlike the parental A20 cells, which are highly immunogenic in immunocompetent $\mathrm{BALB} / \mathrm{c}$ mice but cause a highly aggressive, disseminated and neuroinvasive lymphoma in immunocompromised BALB/c mice, clone E12E is less immunogenic but highly tumorigenic in the liver. While A20 displays a diploid karyotype with a modal number of 40 chromosomes (range 36-41), clone E12E displays a tetraploid karyotype with a broad range in chromosome numbers, from 73 to 90 (Fig. 2a). E12E cells express MHC class I molecules, the death receptor Fas (CD95) and the B cell marker CD45R/B220 (Fig. 2b), but are negative for a panel of other markers tested, including CD markers $5,11 \mathrm{~b}$, 25, 27, 44, 62L, 69, 80, and 117. Although E12E expresses Fas, agonistic anti-Fas antibody did not significantly trigger
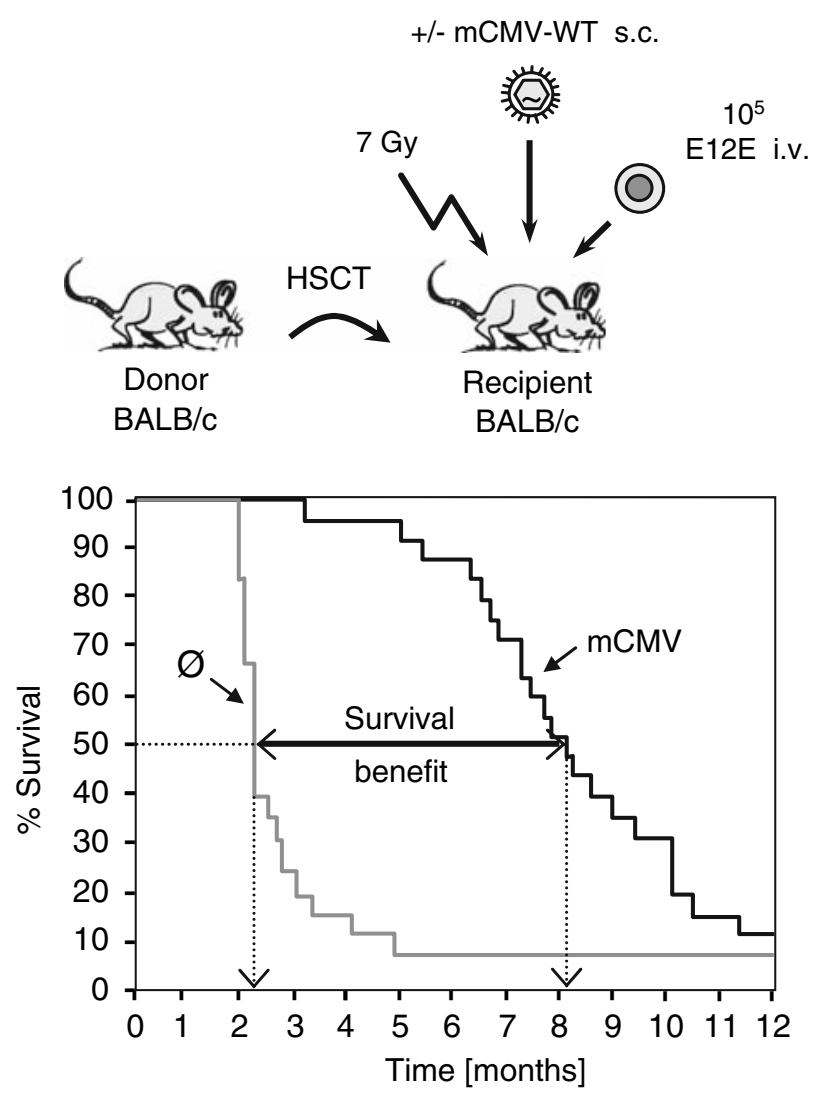

Fig. 1 Experimental tumor model and survival benefit from mCMV infection. (Top) Schema of the experimental setting. Total body $\gamma$-irradiation of $\mathrm{BALB} / \mathrm{c}$ recipients with a single dose of $7 \mathrm{~Gy}$ was performed on day -1. A20 lymphoma cells, liver-adapted clonal variant E12E, were administered intravenously (i.v.) at a dose of $10^{5}$ cells in a mixture with $10^{7} \mathrm{BALB} / \mathrm{c}$-derived femoral and tibial bone marrow cells on day 0. HSCT hematopoietic stem cell transplantation. Subcutaneous (s.c. intraplantar) infection in the left hind footpad was performed $\sim 1-$ $2 \mathrm{~h}$ later with $10^{5}$ plaque forming units of wild-type mCMV, mCMVWT strain Smith. (Bottom) Survival was monitored over a period of 12 months for 25 recipients per group. Shown are Kaplan-Meier plots with black and grey lines representing infected (mCMV) and uninfected $(\varnothing)$ groups, respectively. Dotted lines mark the median survival times. Reproduced from [25] with modifications

apoptosis of E12E cells in cell culture (K.C.E., unpublished data). As shown previously [24], E12E is resistant to tumor necrosis factor TNF- $\alpha$ both in cell culture as well as in vivo.

It is essential to note that E12E is not permissive for mCMV infection and does not even express the immediateearly protein IE1, as evidenced by the absence of intranuclear IE1 protein and resistance to lysis by an IE1-specific cytolytic CD8 T cell line [25], so that the trivial antitumoral mechanism of cytopathic infection of the tumor cells was excluded by virtue of experimental model design. In addition, pre-incubation of E12E with either infectious or UVinactivated mCMV did neither interfere with tumor cell growth in cell culture nor with in vivo tumorigenicity in the target-organ liver, which proved that signaling to tumor cells or occupation of tumor cell surface receptors by the 
Fig. 2 Characterization of the liver-adapted clonal variant E12E of B-cell lymphoma A20. a Comparative karyotype analysis of the BALB/c-derived diploid parental B-cell lymphoma line A20 and its tetraploid clonal variant E12E. Bar markers represent $10 \mu \mathrm{m}$ b Cytofluorometric cell surface phenotyping of clone E12E. Expression of major histocompatibility complex (MHC) class I molecules is representatively shown for molecule $\mathrm{K}^{\mathrm{d}}$, with expression also found for $\mathrm{D}^{\mathrm{d}}$ and $\mathrm{L}^{\mathrm{d}}$. Dotted lines mark the fluorescences measured with the respective FITC-labeled isotype control antibodies a
A20

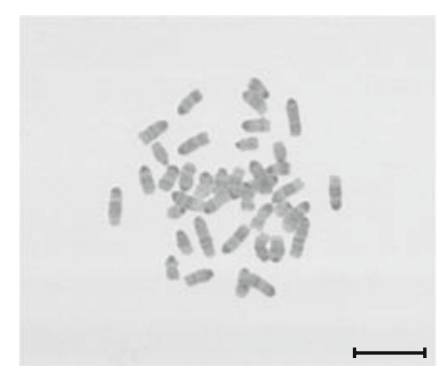

E12E

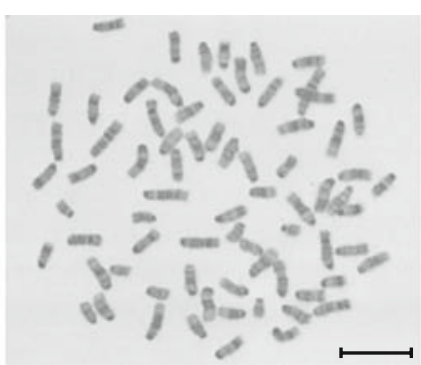

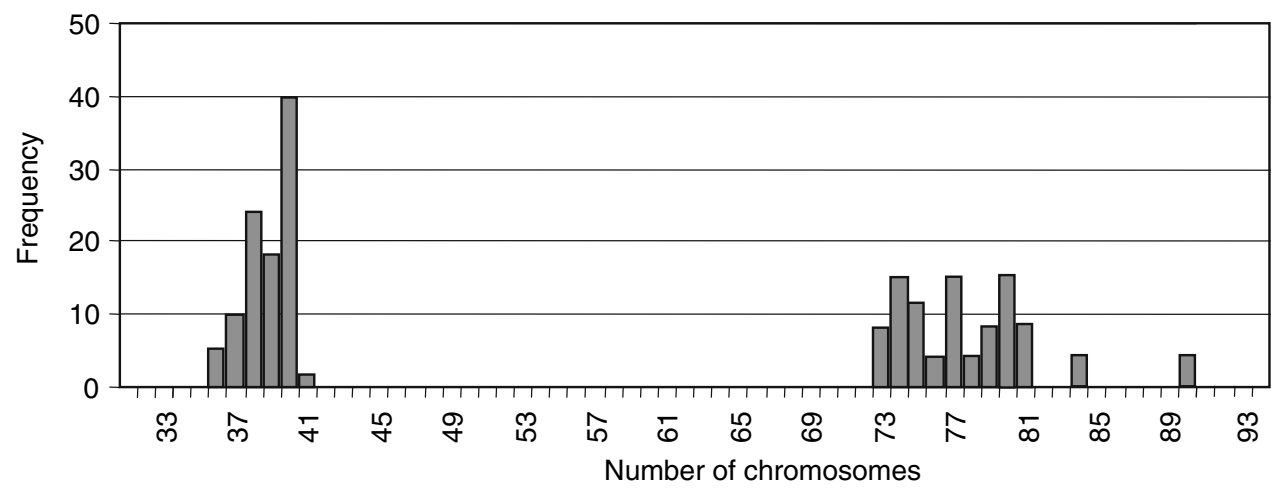

b

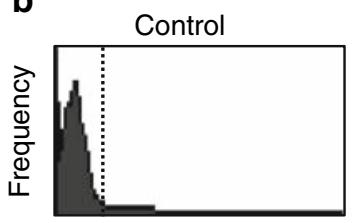

$\alpha-\mathrm{MHC}$ I $\left(\mathrm{K}^{\mathrm{d}}\right)$

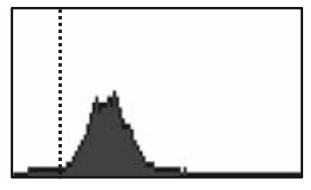

$\alpha-$ Fas

$\alpha-C D 45 R$
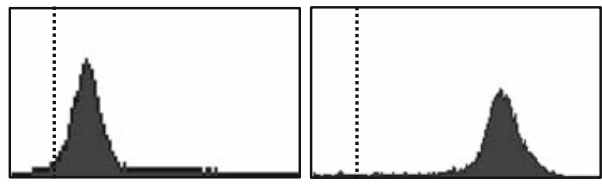

FITC fluorescence intensity binding of virion envelope proteins played no role in this model. In the basic protocol, hematoablative treatment of HSCT recipients by total-body $7 \mathrm{~Gy} \gamma$-irradiation was performed on day -1 , hematopoietic cells and E12E lymphoma cells were administered on day 0 in a mixture, and subcutaneous, intraplantar infection with $10^{5} \mathrm{PFU}$ of mCMV was performed shortly thereafter.

A very basic question that we addressed first was if E12E cells really transmigrate through the discontinuous sinusoidal endothelium to establish lymphoma colonies within liver parenchyma or, alternatively, grow within the sinusoidal space. Silver staining of liver tissue clearly demonstrates an extra-vascular growth of the lymphoma (Fig. 3), which implies that transmigration from the sinusoidal compartment into the liver parenchyma is a critical step in the establishment of E12E lymphoma colonies, a step that might be targeted by CMV infection.

\section{Lymphoma control and survival benefit in the murine model of CMV infection after HSCT}

The dose of bone marrow cells, namely $10^{7}$ cells, was deliberately chosen to allow successful bone marrow recon-

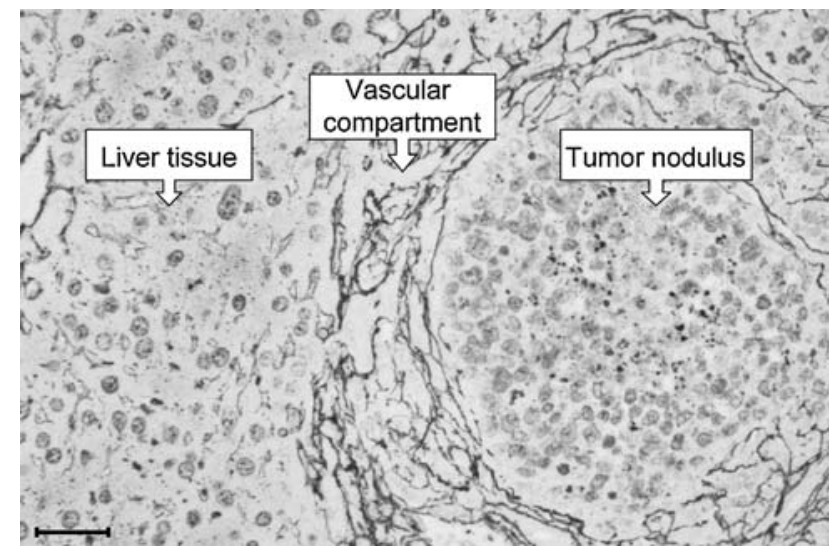

Fig. 3 Extravascular growth of the E12E lymphoma. Liver tissue section taken on day 28 after HSCT and i.v. administration of $10^{6} \mathrm{E} 12 \mathrm{E}$ cells stained by Gomori's silver impregnation method for visualizing the vascular reticulum of blood vessels and sinusoidal endothelium. The bar marker represents $50 \mu \mathrm{m}$

stitution preventing any lethality from mCMV infection itself. In the absence of infection, $10^{5}$ lymphoma cells led to death of $50 \%$ of the recipients within $\sim 2$ months, and less than $10 \%$ of the recipients survived long term, which indicated that immunological reconstitution by HSCT, although being efficient in controlling the virus, was 
insufficient in controlling the lymphoma. It must be considered, however, that in this model a GvL effect is based entirely on non-polymorphic leukemia antigens expressed by E12E. It was all the more surprising then to find a survival benefit of $\sim 6$ months by a simultaneous intraplantar infection with a moderate dose of $10^{5} \mathrm{PFU}$ of mCMV (Fig. 1) [25], which is a very significant period in the life span of mice. Needless to emphasize that the dose of tumor cells has an impact on both the lethality of the tumor and the survival benefit conferred by the infection. At the low dose of $10^{4} \mathrm{E} 12 \mathrm{E}$ cells, which is a closer correlate to the clinical situation of MRD/L (see Introduction), $\sim 50 \%$ of the recipients survived long term, and the $50 \%$ survival benefit was $>8$ months [25].

Besides the survival benefit, the tumor-repressive effect of mCMV infection is also impressively documented by liver macroscopy and by quantitative tumor-specific CD45R immunohistology showing massive tumor manifestation in the absence of infection and only few and small tumor noduli in the presence of infection (Fig. 4). Importantly, the tumor-repressive effect of mCMV infection turned out to be completely independent of HSCT in that it operated also after hematoablative high-dose $\gamma$-irradiation, suggesting that innate or adaptive immune responses mediated either directly by transplanted bone marrow cells or later by their differentiated, mature progeny are not critically involved in the mechanism of E12E lymphoma control.

Similarly important is the finding that E12E lymphoma control is not mediated nonspecifically by any virusinduced inflammatory process or any "viral disease syndrome", for instance, by hyperthermia, hypoxemia, withdrawal of nutrients and/or growth-promoting cytokines, systemic burst of death ligands, or by endothelial injury interfering with tumor cell extravasation/metastasis. This was concluded from the finding that a panel of $\alpha$-herpesviruses HSV-1 and HSV-2, representing strains of broadly different pathogenicity, did not control the E12E lymphoma under otherwise identical experimental conditions. Notably, this included HSV-1 strains JES and KOS as well as HSV-2 strain $\mathrm{ER}^{-}$, which disseminate to and productively infect the liver, the target organ of E12E ([25], J.P., unpublished data). This indicated that destruction of the stromal bed for tumor cell homing by cytopathic infection of the liver cells is unlikely to be the mechanism of tumor control in this organ.

It was certainly a critical question if this tumor-repressive effect of mCMV infection is restricted to B cell lymphomas, or possibly even to the particular, liver-adapted clonal variant E12E of the A20 lymphoma. In the light of the reports on a tumor-repressive effect of hCMV on adult T-cell leukemia/lymphoma in humans, it was an important finding that mCMV infection also controls the murine $\mathrm{T}$ cell lymphoma Esb-MP and its highly aggressive variant Esb-L-CI [25].

\section{Control of the E12E lymphoma operates from a distance}

Since bone marrow cells and their progeny are apparently not involved in the tumor-repressive mechanism, the model was modified by omitting the HSCT, thereby focusing on early events. The lymphoma-repressive effect was found to require viral replication at the local, plantar site of infection, since UV-inactivated mCMV-Smith and the in vivo highly attenuated enhancerless mutant mCMV- $\Delta$ MIEenh [26] were not effective. Similarly, hCMV strain Towne also did not replicate due to the species-specificity of CMVs [25].

Particularly informative were the findings obtained with mCMV- $\Delta \mathrm{M} 36$, a virus lacking the anti-apoptotic viral protein M36 [27, 28]. Probably due to the apoptosis described for infected macrophages [27], this virus is deficient in disseminating from a local site of virus replication to distant target organs, thus failing to infect the liver [25, 29]. Nonetheless, this mutant was still able to repress E12E growth in the liver [25].

This gives us two messages: first, infection of the liver and thus a putative destruction of the stromal bed of the tumor is not the mechanism of tumor control and, second, mCMV operates from a distance, suggesting an involvement of systemic mediators either targeting the tumor cells directly or activating cellular effector cells operating at the site to where the tumor localizes.

\section{Lymphoma control by a transient phase of tumor cell apoptosis in the liver}

A central question was if the unknown, mCMV-induced mediator(s) already prevented the extravasation of the lymphoma cells from the circulation into the target organ, the early homing in the target tissue, or, later on, the growth rate of the tumor cells within the target organ.

These questions were addressed by immunohistological quantification of liver-resident E12E cells in the time course (Fig. 5a) [25]. The first two possible modes of action were clearly excluded by the lymphoma growth curves. In both the infected and the uninfected group E12E cells entered the liver parenchyma from the blood stream through the liver endothelium with the same transmigration rate, though only one out of 40 lymphoma cells was successful in that respect [25], and proliferated in the liver parenchyma until day 2 with the same doubling time of $\sim 26 \mathrm{~h}$, which implies that colonies can reach the 4-cell 
Fig. 4 Lymphoma imaging and computer-assisted quantitation. a1, b1 Lymphoma-repressive effect of mCMV infection in the HSCT setting, demonstrated by liver macroscopy on day 28 . Tumor nodules become prominent by their hyaloid appearance. Bar markers represent $5 \mathrm{~mm}$. Reproduced from [24]. a2, b2 Immunohistological visualization of tumor colonies in liver tissue sections stained for expression of the E12E cell surface marker CD45R (see Fig. 2b). The analysis was performed on day 12 according to the experimental protocol shown in Fig. 1, except that no HSCT was performed and that the initial tumor cell dose was raised to $10^{6} \mathrm{E} 12 \mathrm{E}$ cells. Bar markers represent $500 \mu \mathrm{m}$. Reproduced from [25]. a3, b3 Computer-assisted, colorcoded definition of tumor colony-size classes for the quantitation of tumor burden by sizefrequency diagrams applied to the CD45R-stained liver tissue sections shown in panels $\mathbf{a} 2$ and b2. Percentages in the diagrams indicate the tumor burden in \% of liver tissue section area. See [25] for more detailed information

\section{$\mathrm{E} 12 \mathrm{E}$}
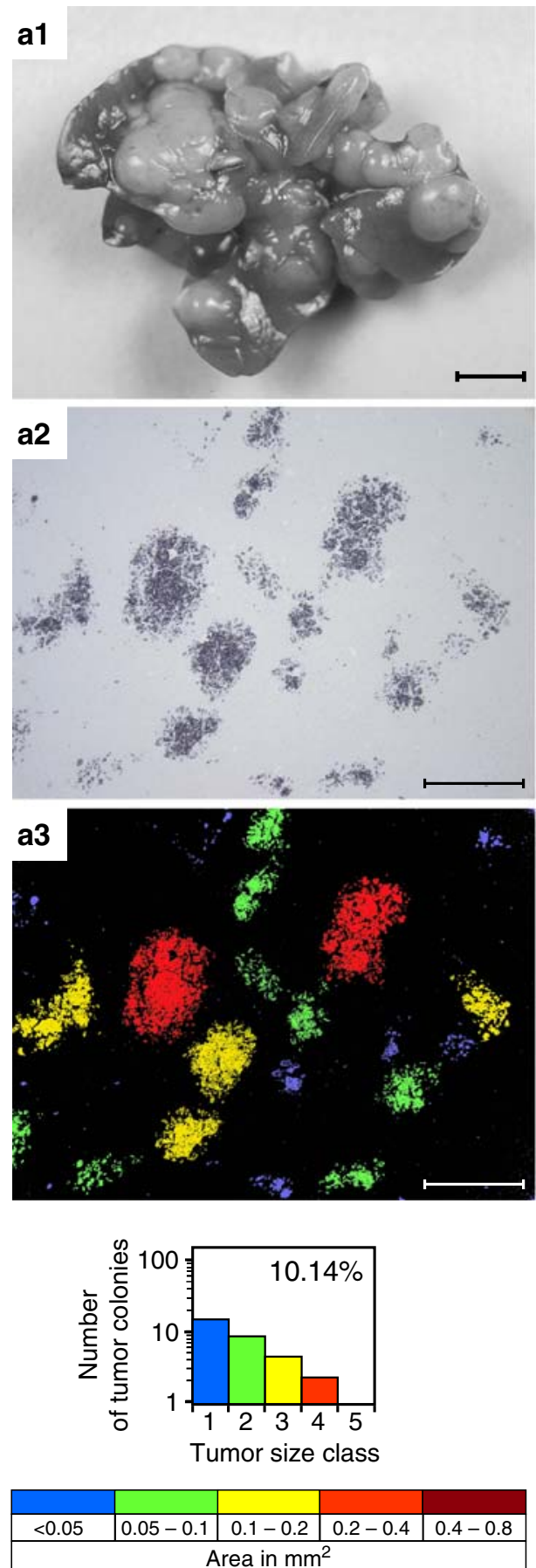

\section{E12E \& mCMV}
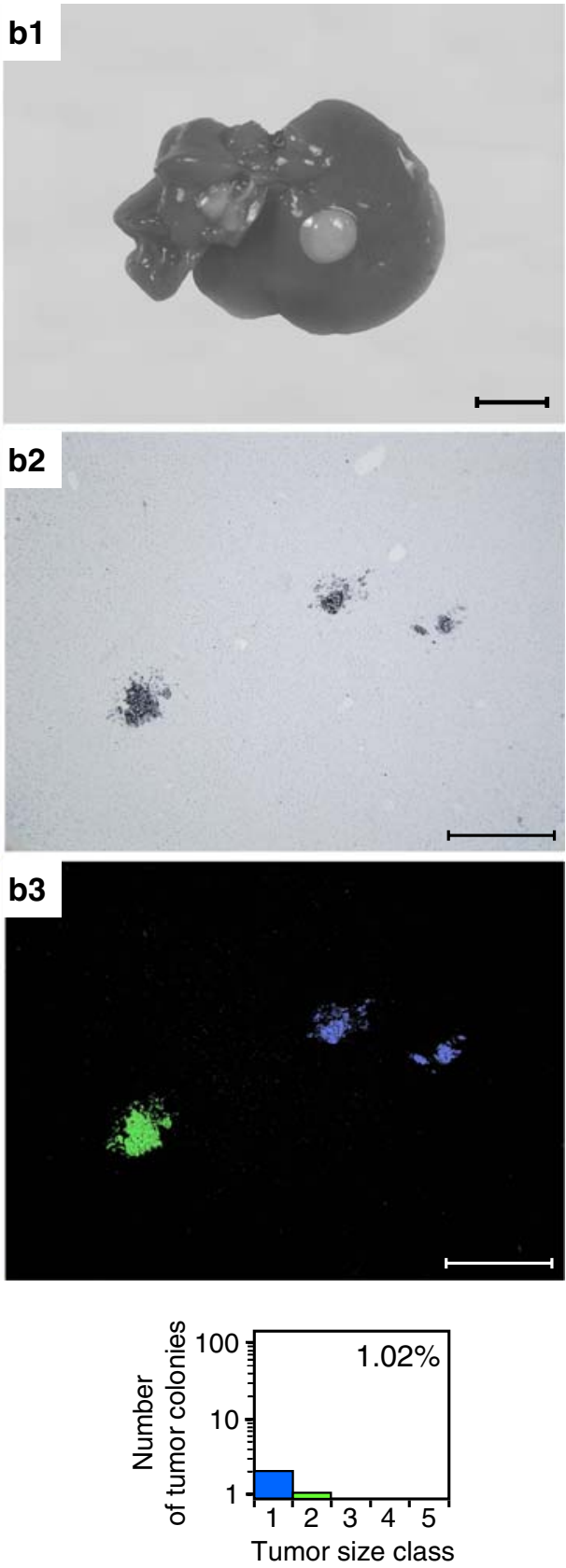

\begin{tabular}{|l|l|l|l|l|}
\hline & & & & \\
\hline$<0.05$ & $0.05-0.1$ & $0.1-0.2$ & $0.2-0.4$ & $0.4-0.8$ \\
\hline \multicolumn{5}{|c|}{ Area in $\mathrm{mm}^{2}$} \\
\hline
\end{tabular}

stage. We refer to this as phase I (Figs. 5a, 6). However, between days 2 and 5, referred to as phase II, the number of lymphoma cells declined in a log-linear fashion specifically in the infected group, whereas exponential growth continued in the uninfected group, which results in the dramatic absolute difference in tumor burden documented above (Fig. 4). Surprisingly, however, this "crisis" was overcome beyond day 5 , and in phase III the lymphoma cells restarted to grow exponentially with an unaltered doubling time.
Thus, infection does not influence the doubling time of the tumor cells but instead interrupts tumor growth transiently.

Interestingly, the phase II crisis clearly precedes mCMV infection of the liver, which becomes apparent only from day 6 onward during phase III (Fig. 5a) [25]. Thus virus replication at the target site of the tumor does not explain the transient lymphoma remission, which is compatible with the fact that E12E cells are not permissive for mCMV infection. Moreover, in phase III, tumor relapse occurred in 
Fig. 5 Time course of E12E growth and apoptotic crisis of the lymphoma after infection. a Lymphoma growth curves $\left(10^{6}\right.$ E12E cells with no HSCT) in presence (red) or absence (black) of infection, displayed as log-linear regression lines with four individual recipients analyzed per time point. The left-hand ordinate scale represents numbers of tumor cells detected in representative liver tissue section areas of $50 \mathrm{~mm}^{2}$. Right-hand ordinate scale and red-rimmed bars represent the numbers of infected hepatocytes present in the corresponding tissue section areas of the four individual recipients analyzed per time point in the infected group. The triphasic E12E growth in the infected group is pointed out by defining phase I (initial growth), phase II (remission), and phase III (relapse). b Corresponding kinetics of lymphoma cell apoptosis in presence (red) or absence (black) of infection. Bars represent the median value numbers of apoptotic cells present in $50-\mathrm{mm}^{2}$ areas of liver tissue sections. Error bars indicate the range observed for the same four individual mice tested per group and time point already for the data shown above (panel a). c Demonstration of selective apoptosis of E12E lymphoma cells present in liver tissue during tumor remission in phase II. Apoptotic tumor cells are identified by two-color immunohistological staining of the lymphoma cell surface marker CD45R (black) and the intracellular apoptosis marker active caspase 3 (red). Note the absence of apoptotic liver cells in the surrounding tissue. IHC immunohistochemistry. The bar marker represents $50 \mu \mathrm{m}$. Reproduced from [25] with rearrangement and modifications

the face of cytopathic liver infection and despite progressive liver tissue destruction [25], which implies that the intrahepatic environment is not influenced in a way that would no longer support lymphoma growth.

The mechanism of the transient remission was elucidated by two-color immunohistology identifying apoptotic E12E lymphoma cells in liver tissue by simultaneous staining for cell surface CD45R and active caspase 3. Quantitation of the apoptotic tumor cells revealed a peak of apoptotic activity precisely coinciding with tumor remission in phase II (Fig. 5b) [25]. It is important to note that the apoptosis was tumor-selective in that only lymphoma cells and not hepatocytes became apoptotic (Fig. 5c).

The timing of infection turned out to be decisive. Beyond day 3 after transfer of the tumor cells, tumor colonies are no longer susceptible to the mCMV-triggered apoptosis-inducing signal. As two further days are required for generating this signal after distant intraplantar infection, that is to enter phase II, this resistance refers to a 64-cell stage of established tumor noduli [25]. Resistance against the tumor-repressive effect of mCMV was not caused by selection of apoptosis-resistant clonal variants of E12E, since lymphoma cells recovered in phase III turned out to be susceptible after serial transfer into secondary recipients [25].

We also considered the possibility that tumor relapse in phase III and non-responsiveness of tumor cells beyond day 6 might not reflect resistance of the tumor colonies but rather indicate only transient presence of the pro-apoptotic signal. However, this explanation was refuted by the finding that infection performed 6 days prior to administration
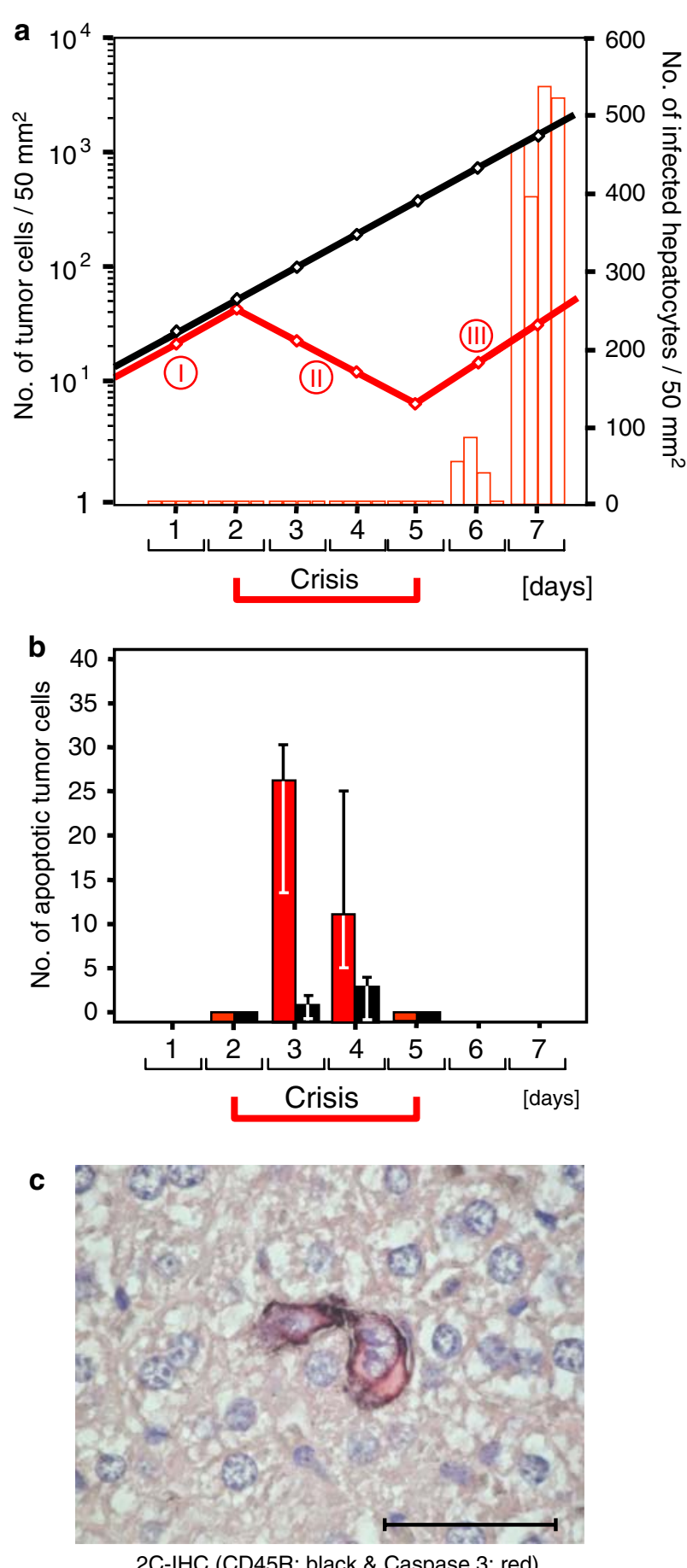

of the lymphoma cells still showed the tumor-repressive function [25].

Interestingly, a quantitative analysis of the number of E12E cells constituting the colonies beyond day 2 revealed the existence of individual colonies with $>4$ cells during phase II [25]. This implies that lymphoma cells still divide during the remission phase; thus, apparently, cell numbers decline because the rate of apoptosis exceeds the growth rate. 
Phase I

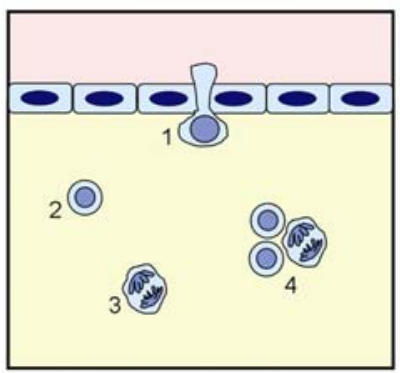

Transmigration \& initial growth
Phase II

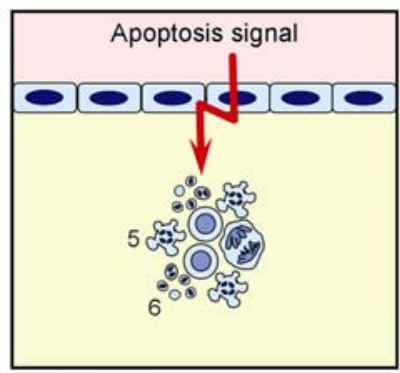

Transient remission
Phase III

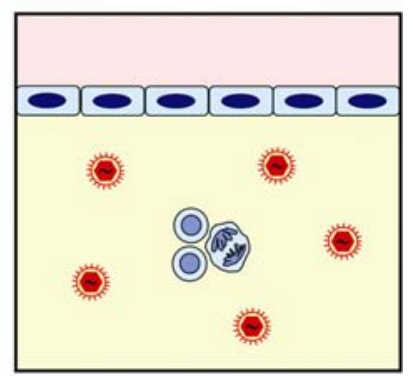

Relapse
Fig. 6 Concluding model of the triphasic lymphoma growth in the presence of infection. Liver sinusoidal endothelial cells separate the vascular compartment (rose) from the intrahepatic compartment (yellow). Lymphoma cells are pictured in stages of endothelium transmi-

It should be emphasized that phase III relapse does not inevitably occur but depends on the initial lymphoma cell load. At a low load, phase II apoptosis can lead to tumor clearance. Our current view explaining the triphasic growth of the lymphoma after infection is sketched in Fig. 6.

\section{Activation of hepatic natural killer cells by distant mCMV infection}

Although, as shown above, mCMV infection operates from a distance, the apoptosis-inducing signal is not necessarily a soluble death ligand encoded by mCMV or induced in the infected cells. Double-chamber cell culture experiments have so far not revealed a soluble factor secreted from infected cells in the lower chamber and causing apoptosis of E12E cells in the upper chamber (K.C.E., unpublished data). Since so far only murine embryonic fibroblasts were tested as infected cells, clearly, these experiments need to be extended to other cell types before a more definite conclusion can be drawn. Alternatively, cytokines known to be induced by a local mCMV infection might induce natural killer (NK) cells located at a distant site. This should not be mixed up with the local NK evasion of infected cells by downregulation of ligands of activating NK cell receptors (reviewed by Lenac et al. in this issue of MMI). Specifically, IFN- $\alpha / \beta$ and interleukin- 12 systemically activate NK cells for cytolytic activity and IFN- $\gamma$ secretion, respectively [30]. Since NK cells are relatively resistant to $\gamma$-irradiation, residual NK cells activated by $\mathrm{CMV}$-induced cytokines might be cellular mediators of the tumor-repressive effect. Though in the first approach NK cells derived from draining lymph nodes of infected, immunocompetent BALB/c mice did not exert a tumor-repressive effect upon adoptive transfer [25], a role for intrahepatic NK cells, the so-called pit cells, was not formally excluded in the lymphoma model in immunocompromised mice. gration (1), interphase (2), mitosis (3), small colonies (4), and apoptosis (5) or disintegrated into apoptotic bodies/vesicles (6).The red virus symbols indicate productive infection of liver parenchyma. Reproduced from [25]

Here we have revisited this issue by studying the activation of hepatic $\mathrm{CD} 49 \mathrm{~b}^{+} \mathrm{NKG} 2 \mathrm{D}^{+} \mathrm{TCR} \beta$ chain-negative NK cells in the time course after $\mathrm{mCMV}$ infection and administration of the E12E lymphoma (Fig. 7). Whereas hepatic NK cells were not induced by the lymphoma alone, mCMV infection led to a significant proportion of NK cells on day 6 in the isolated non-parenchymal fraction of the liver cells (Figs. 7a, 8). Notably, mCMV alone was sufficient for this activation, as there existed no significant difference $(P=0.13$, two-tailed $)$ in hepatic NK cell induction between infected mice carrying or not carrying the lymphoma. Likewise, there was also no significant difference in splenic NK

Fig. 7 Recruitment of hepatic natural killer cells by mCMV infection. a Experimental protocol for the cytofluorometric quantitation of natural killer (NK) cells in liver tissue. Recipient mice were treated as shown in Fig. 1, except that no HSCT was performed and that $10^{6}$ E12E cells were administered. After liver perfusion to remove intravascular cells followed by dissociation of liver tissue, non-parenchymal liver cells were isolated by continuous OptiPrep ${ }^{\mathrm{TM}}$ density gradient centrifugation removing the hepatocytes. Three-color cytofluorometric/FACS analysis was performed by staining for TCR $\beta$-chain, $\mathrm{CD} 49 \mathrm{~b}$, and NKG2D with an electronic gate set on TCR $\beta$-chain ${ }^{+}$cells to exclude $\mathrm{T}$ cells from the analysis. Two-parameter dot plots show the expression of CD49b and NKG2D by the TCR $\beta$-chain-negative nonparenchymal liver cells. Percentages of CD $49 \mathrm{~b}^{+} \mathrm{NKG}^{-} \mathrm{D}^{+} \mathrm{NK}$ cells are indicated in the upper right quadrants. The analysis of three individual mice of the infected (red) and uninfected (black) group is shown for day 6. b Time course of NK cell activation/recruitment in \% of TCR $\beta$ chain-negative non-parenchymal liver cells for the infected (red dots) and the uninfected (black dots) group. Dots represent three individual mice tested per group and time point. Day 6 data refer to the analysis detailed above (panel a). $\mathbf{c}$ The same data as in panel $\mathbf{b}$, but expressed in absolute cell numbers per liver. d Cytolytic activity of non-parenchymal liver cells derived from the infected (red dots) and the uninfected (black dots) groups measured on day 3 by using E12E lymphoma cells as target cells in a 16-h chromium-release assay at the effector-to-target (E/T) cell ratios indicated. Dots represent replicate assay microcultures. Anti-tumoral cytolytic activity in the infected group was attributed to the liver NK cells by loss of activity after immunomagnetic depletion of $\mathrm{CD}_{4} 9 \mathrm{~b}^{+}$cells (open red circles). Note that $\mathrm{T}$ cells present in the non-parenchymal liver cell fraction do not express CD49b 

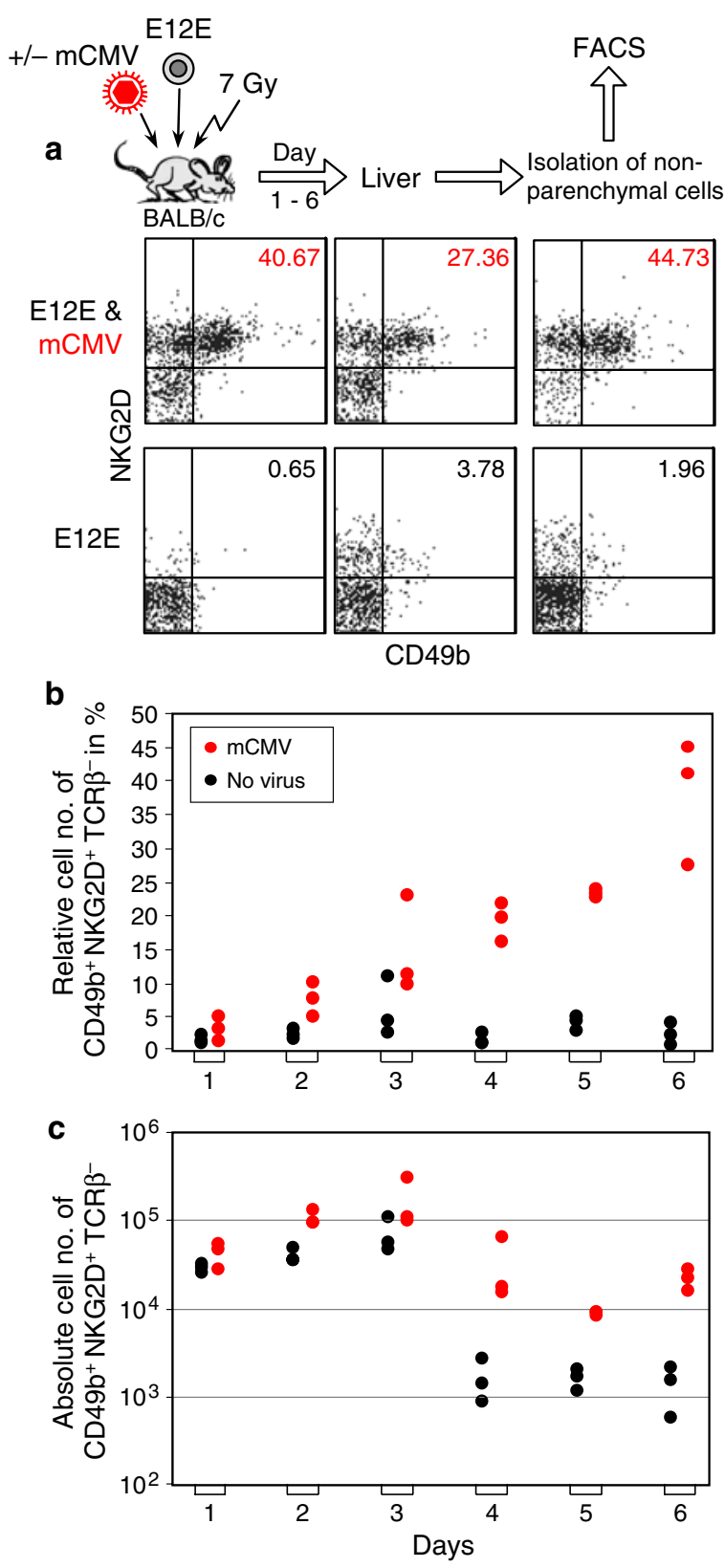

d

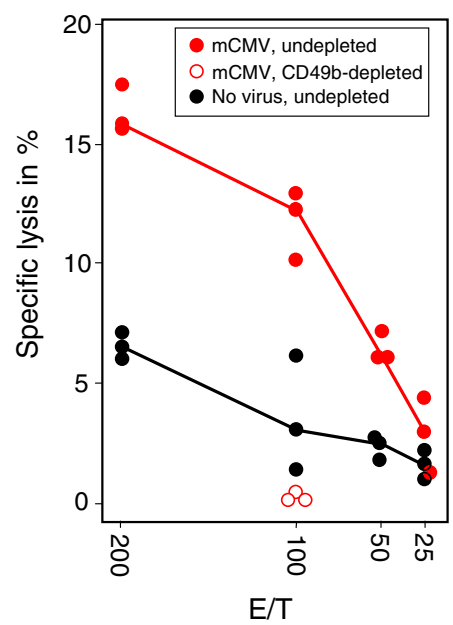

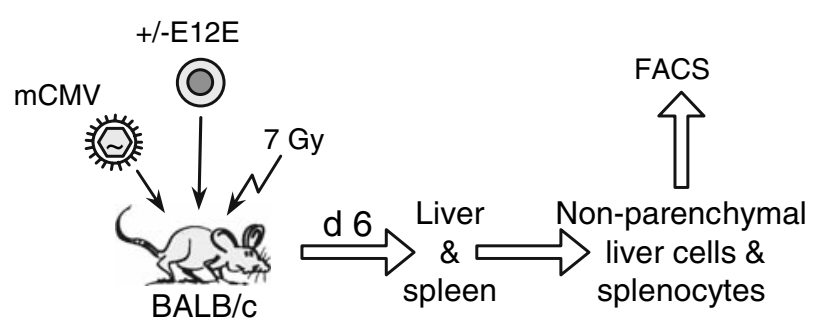

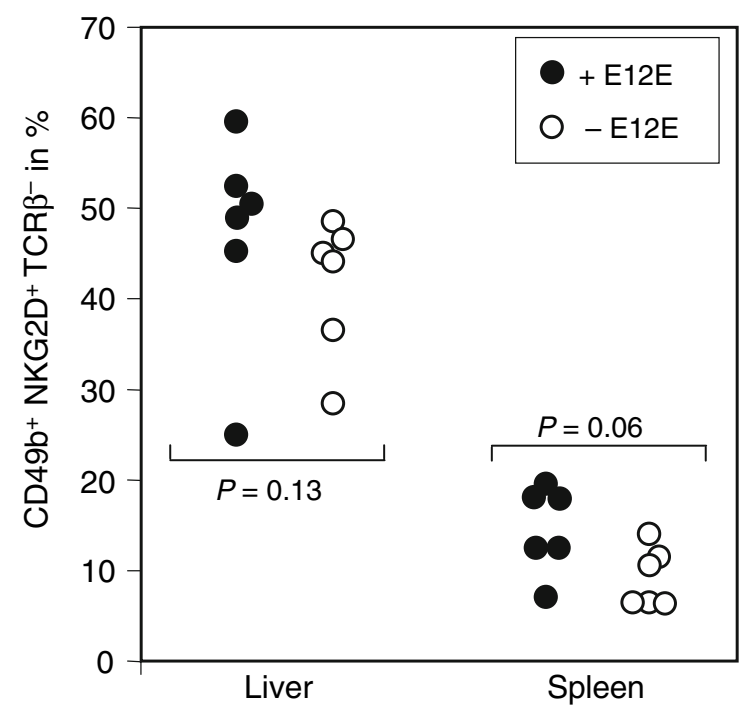

Fig. 8 Lymphoma cells are not required for NK cell recruitment. Top Experimental protocol. See also Fig. 7a. Bottom Percentages of $\mathrm{CD}_{49} \mathrm{~b}^{+} \mathrm{NKG} 2 \mathrm{D}^{+} \mathrm{TCR} \beta$-chain-negative NK cells in the non-parenchymal liver cell fraction as well as in the spleen on day 6 after infection in the presence (black dots) or absence (open circles) of the E12E lymphoma. Symbols represent individual mice tested. For significance analysis, $P$ values were determined by using the distribution-free Wilcoxon-Mann-Whitney rank sum test. Data are not significantly different if $P>0.05$

cell induction $(P=0.06$, two-tailed), although there was a tendency to a somewhat higher activation in the presence of the lymphoma in both liver and spleen (Fig. 8).

As revealed by the kinetics, the proportion of NK cells did not peak during the tumor remission phase II at day 3 (Fig. 5), but instead increased steadily over the observation time (Fig. 7b). Thus, if we assume that the NK cells are the anti-tumoral effector cells, this would suggest resistance of the tumor colonies in the relapse phase II, as discussed in greater detail above. A different explanation, however, is suggested by the absolute numbers of NK cells, which are influenced by the pancytopenia caused by the hematoablative treatment. The absolute number indeed reached its peak on day 3 during the lymphoma remission and abruptly declined thereafter (Fig. 7c). Moreover, non-parenchymal liver cells isolated on day 3 showed cytolytic activity against E12E cells, an activity that was abrogated by immunomagnetic depletion of $\mathrm{CD}_{4} 4 \mathrm{~b}^{+}$cells (Fig. 7d). 

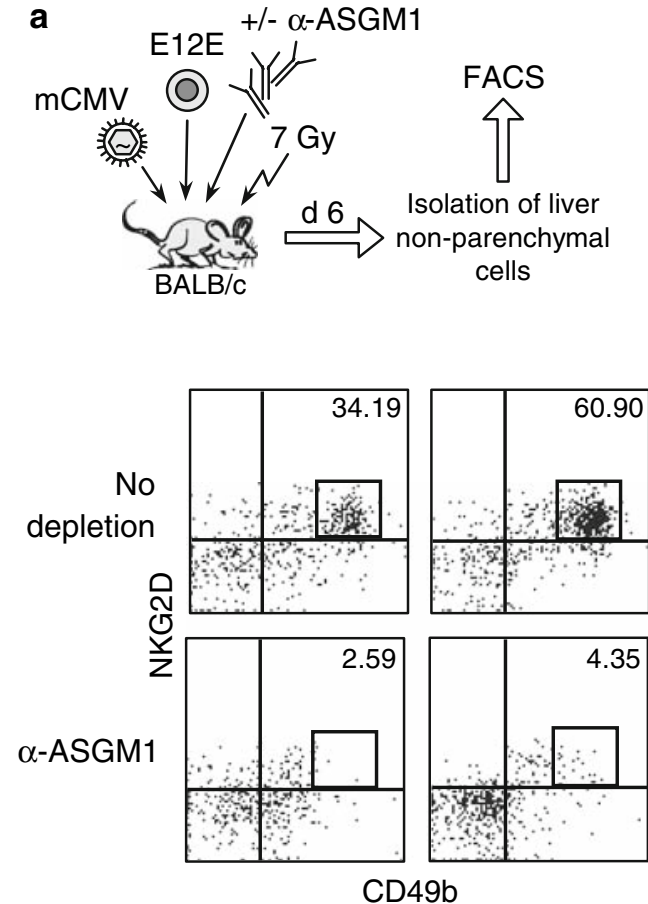

Fig. 9 NK cells are not required for the tumor-repressive effect of mCMV infection. a Cytofluorometric verification of NK cell depletion in the liver. Top Experimental protocol. See also Fig. 7a. For NK cell depletion, antibody directed against asialo GM1 ( $\alpha$-ASGM1 $25 \mu$ i.v., WAKO Chemicals) was administered on days -2 and +2 . Bottom Two-parameter dot plots show the expression of CD49b and NKG2D by the TCR $\beta$-chain-negative non-parenchymal liver cells isolated on day 6 from two individual mice per group. Percentages of

To be precise, the increase in the proportion of NK cells in the liver observed after mCMV infection does not necessarily indicate an activation of liver-resident NK/pit cells. Alternatively, this might also be explained by a recruitment of peripheral NK cells to the liver.

\section{Hepatic NK cells are not critically involved in mCMV- triggered lymphoma control}

Altogether, these findings reasonably argued for hepatic NK cells being the effector cells of the tumor-repressive effect. This hypothesis was directly addressed by in vivo depletion of NK cells using antibodies directed against the ganglioside asialo GM1 ( $\alpha$-ASGM1). This treatment proved to be highly effective in preventing the accumulation of CD $49 \mathrm{~b}^{+} \mathrm{NKG} 2 \mathrm{D}^{+} \mathrm{TCR} \beta$ chain-negative NK cells in the non-parenchymal liver cell fraction of infected BALB/c mice (Fig. 9a).

In the uninfected group, depletion of NK cells with $\alpha$ ASGM1 was found to have only a minor impact on the growth of E12E in the liver. This control experiment made sure that $\alpha$-ASGM1 treatment did not by itself control the

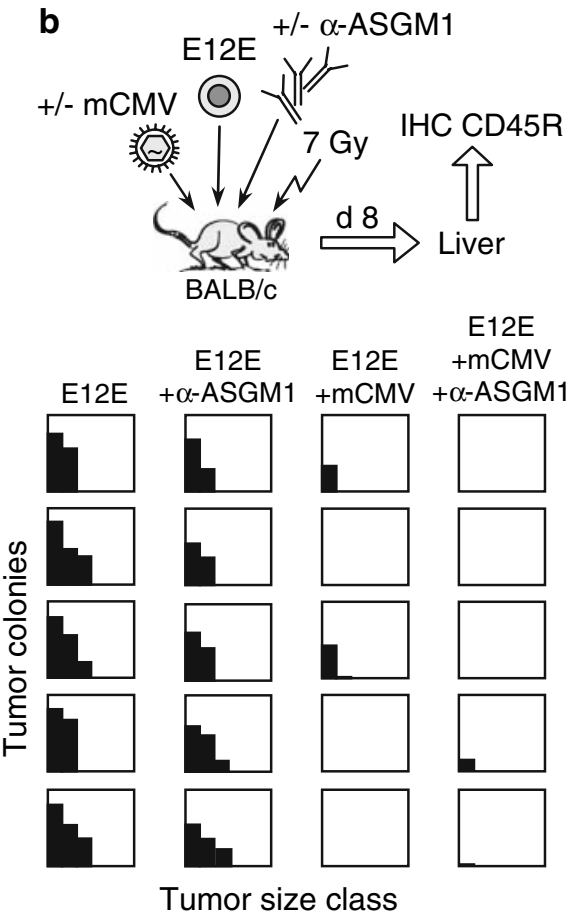

$\mathrm{CD} 49 \mathrm{~b}^{+} \mathrm{NKG} 2 \mathrm{D}^{+} \mathrm{NK}$ cells present in the marked gates are indicated in the upper right quadrants. b Impact of NK cell depletion on E12E lymphoma growth. Top Experimental protocol. IHC Immunohistochemical detection of $\mathrm{CD} 45 \mathrm{R}^{+}$lymphoma cells in liver tissue sections on day 8. Bottom Lymphoma size-frequency diagrams (for definition, see Fig. 4) for five individual mice per group. Group parameters are indicated

lymphoma under the experimental conditions used (Fig. 9b, first two columns). While mCMV infection alone was sufficient to control the lymphoma almost completely, as was expected from all the preceding data, this tumor-repressive effect was not abolished by NK cell depletion with $\alpha$ ASGM1 (Fig. 9b, last two columns). In accordance with the NK-cell depletion data, liver-derived NK cells isolated from infected donor mice failed to control the E12E lymphoma upon adoptive cell transfer into uninfected indicator recipients (K.C.E., data not shown). In conclusion, NK cells are apparently not required for the mCMV-mediated lymphoma control.

\section{Concluding remarks}

The model of coincident infection and intravenous administration of B- or T-cell lymphomas has revealed a novel and unpredicted, lymphoma-repressive function of mCMV. The data have shown that mCMV infection operates from a distance and becomes effective in the target organ of the lymphomas, the liver in the reported cases, before the liver becomes infected and even after peripheral infection with a 
virus mutant genetically deficient in dissemination and thus never infecting the liver. The mechanism of the tumorrepressive function was identified as an induction of lymphoma cell apoptosis, although the death ligand-death receptor pair is still unknown (for more detailed discussion, see [25]). Importantly, as shown here, hepatic NK cells are not required as cellular mediators of the tumor-repressive effect. This was somewhat surprising and, admittedly, also somewhat disappointing, since peripheral mCMV infection induces a relative and absolute enrichment of CD49b ${ }^{+}$NKG2D ${ }^{+}$TCR $\beta$ chain-negative NK cells in the liver and since the B-cell lymphoma E12E is indeed susceptible to lysis by these NK cells. It thus appears now that lymphoma cell apoptosis and induction or liver recruitment of NK cells are coinciding events triggered by mCMV but are not causally linked. One might speculate if possibly the same virally triggered factors are responsible for this coincidence.

It is of importance that the tumor-repressive signals can target the tumor cells after their settlement in the liver, when they are young colonies. However, it is similarly important that advanced colonies, namely colonies of the 32-64 cell stage, lose susceptibility. As a consequence, in $\mathrm{MRD} / \mathrm{L}, \mathrm{mCMV}$ may be able to interrupt tumor relapse at an early stage and it may also interfere with tumor metastasis, but it cannot cause remission of an established tumor.

Are these findings of any importance, besides basic science interest? It is clearly a central question whether or not hCMV has the same lymphoma-repressive property in HSCT patients, but there is no feasible way to find out experimentally. A hint for a beneficial role of hCMV infection has been reported for patients with adult T-cell leukemia/lymphoma (see Introduction), but clinical studies have so far not specifically addressed this question in multi-center trials.

A difficulty in obtaining direct clinical evidence lies in the fact that active virus replication is required at the time when tumor relapse from MRD/L is at an early stage, a condition of coincidence seldom met in the clinical situation of HSCT. Needless to emphasize that hCMV infection is not a therapeutic option. However, the molecular identification of the virally-induced and tumor-selective proapoptotic signal, might open a new possibility to cope with early stages of lymphoma relapse and periods of tumor metastasis.

Acknowledgments We greatly appreciate the help by Jürgen Olert (Institute for Pathology, Mainz) with the karyotype analysis and by Hans Anton Lehr (Institute for Pathology, Mainz) with the Gomori staining of liver tissue. We thank Christof K. Seckert from our institute for help with the isolation of non-parenchymal liver cells. This work was supported by the Deutsche Forschungsgemeinschaft, SFB 432, individual project A10 "Influence of cytomegalovirus infection on the risk of tumor relapse after bone marrow transplantation".

\section{References}

1. Appelbaum FR (2001) Haematopoietic cell transplantation as immunotherapy. Nature 411:385-389

2. Appelbaum FR (2003) The current status of hematopoietic cell transplantation. Annu Rev Med 54:491-512

3. Kolb HJ, Schmid C, Barrett AJ, Schendel DJ (2004) Graft-versusleukemia reactions in allogeneic chimeras. Blood 103:767-776

4. Leung W, Iyengar R, Turner V, Lang P, Bader P, Conn P, Niethammer D, Handgretinger R (2004) Determinants of antileukemia effects of allogeneic NK cells. J Immunol 172:644-650

5. Ruggeri L, Mancusi A, Capanni M, Martelli MF, Velardi A (2005) Exploitation of alloreactive NK cells in adoptive immunotherapy of cancer. Curr Opin Immunol 17:211-217

6. Hebart H, Einsele H (2004) Clinical aspects of CMV infection after stem cell transplantation. Hum Immunol 65:432-436

7. Riddell SR (1995) Pathogenesis of cytomegalovirus pneumonia in immunocompromised hosts. Semin Respir Infect 10:199-208

8. Childs B, Emanuel D (1993) Cytomegalovirus infection and compromise. Exp Hematol 21:198-200

9. Simmons P, Kaushansky K, Torok-Storb B (1990) Mechanisms of cytomegalovirus-mediated myelosuppression: perturbation of stromal cell function versus direct infection of myeloid cells. Proc Natl Acad Sci USA 87:1386-1390

10. Fujiwara H, Matsumoto T, Eizuru Y, Matsushita K, Ohtsubo H, Kukita T, Imaizumi R, Matsumoto M, Hidaka S, Arima N, Tei C (2000) Cytomegalovirus infection is not necessarily a poor prognostic factor in adult T-cell leukemia/lymphoma. J Med Virol 62:140-143

11. Fujiwara H, Eizuru Y, Matsumoto T, Kukita T, Imaizumi R, Kawada H, Ohtsubo H, Matsushita K, Arima N, Tei C (2001) The significance of cytomegalovirus infection over the clinical course of adult T-cell leukemia/lymphoma. Microbiol Immunol 45:97-100

12. Brune W, Wagner M, Messerle M (2006) Manipulating cytomegalovirus genomes by BAC mutagenesis: strategies and applications. In: Reddehase MJ (ed) Cytomegaloviruses: molecular biology and immunology. Caister Academic Press, Norfolk, pp 63-90

13. Holtappels R, Munks WM, Podlech J, Reddehase MJ (2006) CD8 T-cell-based immunotherapy of cytomegalovirus disease in the mouse model of the immunocompromised bone marrow transplantation recipient. In: Reddehase MJ (ed) Cytomegaloviruses: molecular biology and immunology. Caister Academic Press, Norfolk, pp 383-418

14. Shenk T (2006) Human cytomegalovirus genomics. In: Reddehase MJ (ed) Cytomegaloviruses: molecular biology and immunology. Caister Academic Press, Norfolk, pp 49-62

15. Digel M, Sinzger C (2006) Determinants of endothelial cell tropism of human cytomegalovirus. In: Reddehase MJ (ed) Cytomegaloviruses: molecular biology and immunology. Caister Academic Press, Norfolk, pp 445-464

16. Apperley JF, Dowding C, Hibbin J, Buiter J, Matutes E, Sissons PJ, Gordon M, Goldman JM (1989) The effect of cytomegalovirus on hemopoiesis: in vitro evidence for selective infection of marrow stromal cells. Exp Hematol 17:38-45

17. Busch FW, Mutter W, Koszinowski UH, Reddehase MJ (1991) Rescue of myeloid lineage-committed preprogenitor cells from cytomegalovirus-infected bone marrow stroma. J Virol 65:981984

18. Mutter W, Reddehase MJ, Busch FW, Bühring HJ, Koszinowski UH (1988) Failure in generating hemopoietic stem cells is the primary cause of death from cytomegalovirus disease in the immunocompromised host. J Exp Med 167:1645-1658

19. Reddehase MJ (1991) Bone marrow dysfunction in irradiated, cytomegalovirus-infected mice. Transplant Proc 23:10-11 
20. Mayer A, Podlech J, Kurz S, Steffens HP, Maiberger S, Thalmeier K, Angele P, Dreher L, Reddehase MJ (1997) Bone marrow failure by cytomegalovirus is associated with an in vivo deficiency in the expression of essential stromal hemopoietin genes. J Virol 71:4589-4598

21. Steffens HP, Podlech J, Kurz S, Angele P, Dreis D, Reddehase MJ (1998) Cytomegalovirus inhibits the engraftment of donor bone marrow cells by downregulation of hemopoietin gene expression in recipient stroma. J Virol 72:5006-5015

22. Reddehase MJ, Dreher-Stumpp L, Angele P, Balthesen M, Susa M (1992) Hematopoietic stem cell deficiency resulting from cytomegalovirus infection of bone marrow stroma. Ann Hematol 64:A125-A127

23. Mori T, Ando K, Tanaka K, Ikeda Y, Koga Y (1997) Fas-mediated apoptosis of the hematopoietic progenitor cells in mice infected with murine cytomegalovirus. Blood 89:3565-3573

24. Erlach KC, Podlech J, Rojan A, Reddehase MJ (2002) Tumor control in a model of bone marrow transplantation and acute liverinfiltrating B-cell lymphoma: an unpredicted novel function of cytomegalovirus. J Virol 76:2857-2870

25. Erlach KC, Böhm V, Seckert CK, Reddehase MJ, Podlech J (2006) Lymphoma cell apoptosis in the liver induced by distant murine cytomegalovirus infection. J Virol 80:4801-4819
26. Ghazal P, Messerle M, Osborn K, Angulo A (2003) An essential role of the enhancer for murine cytomegalovirus in vivo growth and pathogenesis. J Virol 77:3217-3228

27. Ménard C, Wagner M, Ruzsics Z, Holak K, Brune W, Campbell AE, Koszinowski UH (2003) Role of murine cytomegalovirus US22 gene family members in replication in macrophages. J Virol 77:5557-5570

28. Cicin-Sain L, Ruzsics Z, Podlech J, Bubic I, Ménard C, Jonjic S, Reddehase MJ, Koszinowski UH (2008) Dominant-negative FADD rescues the in vivo fitness of a cytomegalovirus lacking an anti-apoptotic viral gene. J Virol 82:2056-2064

29. Cicin-Sain L, Podlech J, Messerle M, Reddehase MJ, Koszinowski UH (2005) Frequent coinfection of cells explains functional in vivo complementation between cytomegalovirus variants in the multiply infected host. J Virol 79:9492-9502

30. Orange JS, Biron CA (1996) Characterization of early IL-12, IFNalphabeta, and TNF effects on antiviral state and NK cell responses during murine cytomegalovirus infection. J Immunol 156:47464756 